GEOLOGICAL SURVEY CIRCULAR 612

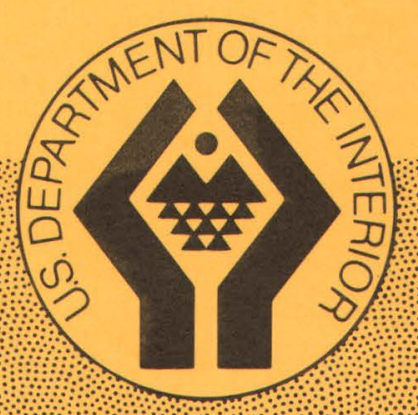

\title{
Gold in Minerals and the \\ Composition of Native Gold
}





\section{Gold in Minerals and the Composition of Native Gold}

By Robert S. Jones and Michael Fleischer

GEOLOGICAL SURVEY CIRCULAR 612

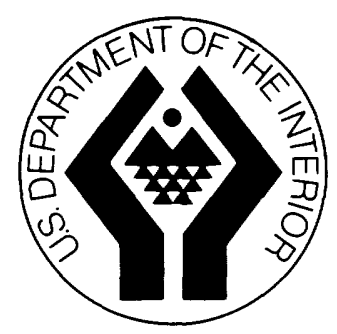


United States Department of the Interior WALTER J. HICKEL, Secretary

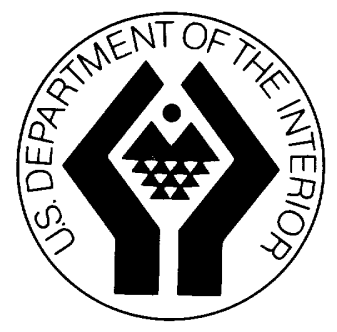

Geological Survey William T. Pecora, Director 


\section{CONTENTS}

$\begin{array}{lr}\text { Abstract } & \text { Page } \\ \text { Introduction } & 1 \\ \text { General geochemical considerations } & 1 \\ \text { Gold in minerals } & \\ \text { Composition and the fineness of gold } & \\ \text { References cited } & \end{array}$

TABLES

TABLE 1. Major gold-bearing minerals

2. Analyses of precious metals in minerals made before $1955 \ldots$

3. Analyses of gold in minerals made since 1954

4. Variation in fineness of gold with depth, Lily mine, Transvaal, South Africa -

5. Fineness of mill bullion prior to 1882 at the Homestake mine, South Dakota _.............. 15 
. 


\title{
GOLD IN MINERALS AND THE COMPOSITION OF NATIVE GOLD
}

\author{
By Robert S. Jones and Michael Fleischer
}

\begin{abstract}
Gold occurs in nature mainly as the metal and as various alloys. It forms complete series of solid solutions with silver, copper, nickel, palladium, and platinum. In association with the platinum metals, gold occurs as free gold as well as in solid solution.

The native elements contain the most gold, followed by the sulfide minerals. Several gold tellurides are known, but no gold selenides have been reported, and only one sulfide, the telluride-sulfide mineral nagyagite, is known.

The nonmetallic minerals carry the least gold, and the light-colored minerals generally contain less gold than the dark minerals.

Some conclusions in the literature are conflicting in regard to the relation of fineness of native gold to its position laterally and vertically within a lode, the nature of the country rocks, and the location and size of nuggets in a streambed, as well as to the variation of fineness within an individual nugget.
\end{abstract}

\section{INTRODUCTION}

This report on the occurrence of gold in minerals and on the fineness of native gold was prepared as background material for the Heavy Metals program of the U.S. Geological Survey, an intensified program of research on new sources of heavy metals, including gold.

\section{GENERAL GEOCHEMICAL CONSIDERATIONS}

Gold belongs to group Ib of the periodic table, as do silver and copper. Its atomic number is 79, and atomic weight is 197.0 ; it consists of a single isotope. Its metallic radius is $1.44 \mathrm{~A}$., univalent ionic radius $1.37 \mathrm{~A}$., and trivalent ionic radius $0.85 \mathrm{~A}$.

Gold is strongly siderophilic and somewhat chalcophilic; that is, it tends to be concentrated in the metallic phase of meteorites, with much lower concentrations in the sulfite phase, and occurs in much lesser amounty in the silicate phase. Gold occurs in natur? mainly as the metal and as various alloys, especially with silver, and as intermetallic compounds. Laboratory studies show that gold can form complete series of solid solutions with silver, copper, nickel, platinum, and palladium. Gold is commonly present in association with platinum metals; most microscopic stucies have shown that free gold is present in platinum, but a recent electron-probe analysis of ferroplatinum shows uniform distributior of gold (Ottemann and Augustithis, 1967). This distribution indicates that gold and platinum are present in solid solution.

Several gold tellurides are known (table 1), but no gold selenides have been repcrted, and the only mineral in which gold is certainly combined with sulfur is the telluride-sulfide, nagyagite. Gold commonly occurs in sulfide minerals, but largely, if not entirely, as the free metal; it is uncertain whether any gold occurs in these minerals in true isomorphous substitution.

It is even less likely that gold is present in ionic substitution in silicate minerals. Mantei and Brownlow (1967) state, "Tle concentration of gold in the various minerals is probably due to an inclusion or entrapping phenomena rather than to ionic substitutior. Because of its oxidation potential, it would 1 ? difficult for gold to become oxidized and thus be able to take part in ionic substitution. Krauskopf (1951) states that simple ionic gold can not exist in geological environments, although complex ions containing gold may form. Ringwood (1955) points out that $\mathrm{Au}^{+}$, because of its 
large electronegativity, would form a very weak covalent bond, and one which would prefer not to form. Thus the gold of a crystallizing magma tends to concentrate in the residual fluids. The factors which would control the amount of gold entrapped in a given mineral would be the concentration of gold in the magma at the time of crystallization and the type of crystal structure formed by the mineral."

Helgeson and Garrels (1968), on the basis of thermodynamic calculations, think that all but marginal or low-grade hydrothermal native gold deposits form above $175^{\circ} \mathrm{C}$ and, at elevated temperatures, most hydrothermal solutions are probably distinctly acid. They believe that gold is present primarily in the form of aurous chloride complexes in contrast to the low-temperature considerations of Krauskopf (1951) and Cloke and Kelly (1964) who suggest that the aqueous species $\mathrm{AuCl}_{4}^{-}$is the principal form of dissolved gold in hydrothermal solutions.

Goni, Guillemin, and Sarcia (1967) have investigated the stability of colloidal suspensions of gold and the formation of nuggets. Stable colloidal suspensions of ionic and even metallic gold can form which can be flocculated to form nuggets. Textures common in gold deposits can be reproduced in gold films formed by the diffusion of gold solutions through silica gel.

Gold occurs in notable amounts in hydrothermal veins and in placer deposits, and to a much lesser extent in pegmatites and contact metamorphic deposits. Common minerals associated with gold in veins are quartz and pyrite. Some other common minerals associated with gold (Lincoln, 1911; Schwartz, 1944) are pyrrhotite, arsenopyrite, chalcopyrite, sphalerite, galena, molybdenite, tellurides, selenides, magnetite, scheelite, feldspar, sericite, biotite, chlorite, amphiboles, garnet, tourmaline, carbonates, and fluorite.

\section{GOLD IN MINERALS}

Minerals that have been reported to contain major amounts of gold are listed on table 1. Many analyses have been made for some of the precious metals in minerals that contain little gold; those made before 1955 are listed in table 2 and those made since 1954 are in table 3 . The more recent analyses have been made by more sensitive methods, mostly by neutron activation, than the older ones, and values as low as 0.0003 ppm (part per million) are reported. However, comparatively few neutron activation analyses of rock-forming minerals have been reported in recent years. The older analyses tend to be significantly higher than more recent analyses of the same minerals. The highest gold values listed in tables 2 and 3 are reported for the native elements; next highest are for the sulfide minerals.

TABLE 1.-Major gold-bearing minerals

Gold

$$
\begin{aligned}
& \text { Au. Cubic, sp gr } 19.3 \text { (pure Au), } \\
& \text { decreasing with ircreasing con- } \\
& \text { tent of Ag. Forms a complete } \\
& \text { series of solid solutions with silver } \\
& \text { (see electrum and silver, below); } \\
& \text { commonly contains 10-15 percent } \\
& \text { Ag. Also reported in percent: Cu } \\
& \text { (max 20.4), Fe (max 0.1), rarely } \\
& \text { Bi (max 2.9), Sn (max 0.3), Pb } \\
& \text { (max 0.2), Zn (max 0.8), Al (max } \\
& \text { 0.10), Mn (max 0.002). See sec- } \\
& \text { tion "Composition and Fineness } \\
& \text { of Gold." }
\end{aligned}
$$

Varieties:

Electrum, $(\mathrm{Au}, \mathrm{Ag})$, argentian gcld with $>\mathbf{2 0}$ percent Ag.

Porpezite, (Au,Pd), palladian go'd with 5-10 percent $P d$.

Rhodite, (Au,Rh), rhodian gold(?) with 34-43 percent Rh.

Auricupride (cuproauride) has ginerally been considered to be a solid solution of gold in copper, near $\mathrm{AuCu}_{3}$ in composition. Ramdohr (1967) states, however, that study of "red gold" from Laksia, Cyprus, has shown the presence of three' distinct phases $\mathrm{Au}-\mathrm{Cu}$ solid solutions, the compound $\mathrm{AuCu}_{3}$ with a characteristic violet color, and the compound $\mathrm{AuCu}$.

Aurosmirid, aurosmiridium (=aurian osmiridium) $\mathrm{Au} 19.3$ percent. Probably a mixture containing gold.

(Palache and others, 1944, p. 111).

Silver

( $\mathrm{Ag}, \mathrm{Au})$. Aurian silver, with $0-50$ percent Au.

Küstelite = aurian silver.

Gold-amalgam

$\mathrm{Au}_{2} \mathrm{Hg}_{3}$ (?)

Au 34.2-41.6 percent.

Maldonite

$\mathrm{Au}_{2} \mathrm{Bi}$. Cubic.

$\mathrm{Au}$ 64.5-65.1 percert; $\mathrm{Bi} 34.9$ 35.5 percent.

Aurostibite

$\mathrm{AuSb}_{2}$. Cubic, pyrite tyre.

Au 43.5-50.9 percent.

Krennerite

$\mathrm{AuTe}_{2}$. Orthorhombic.

Müllerine $=$ Krennerite Au 30.7-43.9 percent.

Speculite $=$ Krennerite $(?)$ 
TABLE 1.-Major gold-bearing minerals-Continued

Calaverite

AuTe. Monoclinic.

Au 39.2-42.8 percent.

Coolgardite $=\mathbf{a}$ mixture of calaverite, coloradoite, and sylvanite.

Sylvanite

(Au,Ag) $\mathrm{Te}_{2}$, with $\mathrm{Au}: \mathrm{Ag}$ usually nearly $1: 1$, that is, $\mathrm{AgAuTe}_{4}$. Monoclinic.

Au 24.25-29.9 percent.

Goldschmidtite $=$ Sylvanite.

Kostovite $\mathrm{CuAuTe}_{4}$

Au 25.2 percent (Terziev, 1966).

Petzite

$\mathrm{Ag}_{3} \mathrm{AuTe}_{2}$

Au 19.0-25.2 percent.

Antamokite $=$ a mixture of petzite and altaite.
TABLE 1.-Major gold-bearing minerals-Concluded

Hessite

$\mathrm{Ag}_{2}$ Te. Monoclinic, pseudocubic. Reported to contain as much as 4.7 percent $\mathrm{Au}$.

Montbrayite $\quad \mathrm{Au}_{2} \mathrm{Te}_{3}$. Triclinic.

Au 38.6-44.3 percent.

Nagyagite $\quad \mathrm{Pb}_{5} \mathrm{Au}(\mathrm{Te}, \mathrm{Sb})_{4} \mathrm{~S}_{5-8}(?)$.

Monoclinic (?).

Au 7.4-10.2 percent.

Silberphyllinglanz = Nagyagite .

Blatterine = Nagyagite.

Aurobismuthinite ( $\mathrm{Bi}, \mathrm{Au}, \mathrm{Ag})_{5} \mathrm{~S}_{6}(?)$

$\mathrm{Au} 12.3$ percent; Aq 2.3 percent. Probably a mixture (Palache and others, 1944 p. 278).

TABLE 2.-Analyses of precious metals in minerals made before 1955

[Minerals containing higher amounts of gold are listed in table 1. N. D., not determined]

\begin{tabular}{|c|c|c|c|c|c|}
\hline Mineral and locality & $\begin{array}{c}\text { Gold } \\
(\mathrm{ppm})\end{array}$ & $\begin{array}{c}\text { Silver } \\
(\mathrm{ppm})\end{array}$ & $\underset{\text { (ppm) }}{\text { Platinum }}$ & Remarks & Reference \\
\hline \multicolumn{6}{|c|}{ Elements } \\
\hline $\begin{array}{l}\text { Arsenic, As } \\
\text { Germany, Andreasberg, Harz }\end{array}$ & 150 & $>1,000$ & 20 & One sample -- & $\begin{array}{l}\text { Noddack and } \\
\text { Notdack (1931). }\end{array}$ \\
\hline $\begin{array}{l}\text { Copper, Cu } \\
\quad \text { Norway, Kviteseid }\end{array}$ & 200 & $>1,000$ & .3 & Two samples - & 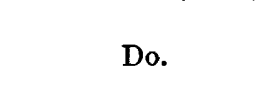 \\
\hline $\begin{array}{l}\text { Iridosmine, (Os,Ir) } \\
\quad \text { Australia, New South Wales }\end{array}$ & 800 & ---- & $>1,000$ & Eight samples & Do. \\
\hline $\begin{array}{l}\text { U.S.S.R., Urals } \\
\text { Iron, (Fe,Ni) }\end{array}$ & 1,000 & 600 & $>1,000$ & ----------- & Do. \\
\hline Greenland, Ovifak, Disko & $1-5$ & 5-10 & 5 & In basalt & $\begin{array}{l}\text { Goldschmidt and } \\
\text { Peters (1932). }\end{array}$ \\
\hline $\begin{array}{l}\text { United States: } \\
\text { Canyon Diablo, Ariz } \\
\text { Holbrook, Ariz }\end{array}$ & $\begin{array}{r}5 \\
10\end{array}$ & $\begin{array}{l}5 \\
1\end{array}$ & $\begin{array}{l}10-100 \\
10-100\end{array}$ & In meteorite - & $\begin{array}{l}\text { Do. } \\
\text { Do. }\end{array}$ \\
\hline $\begin{array}{l}\text { Mexico } \\
\text { Coahuila - } \\
\text { Chile, Corrizatillo }\end{array}$ & $\begin{array}{r}1-5 \\
5-10\end{array}$ & $\begin{array}{r}5 \\
5-10\end{array}$ & $\begin{array}{r}10-100 \\
10\end{array}$ & In meteorite - & $\begin{array}{l}\text { Do. } \\
\text { Do. }\end{array}$ \\
\hline $\begin{array}{l}\text { Germany, Bühl, near Kassel } \\
\text { Czechoslovakia, Knyahinya } \\
\text { Portugal, Sao Juliao de Moreira }\end{array}$ & $\begin{array}{l}.5 \\
10 \\
10\end{array}$ & $\begin{array}{r}5-10 \\
1 \\
5\end{array}$ & $\begin{array}{l}.2 \\
10-100 \\
10-100\end{array}$ & $\begin{array}{l}\text { In basalt ---- } \\
\text { In meteorite - }\end{array}$ & $\begin{array}{l}\text { Do. } \\
\text { Do. } \\
\text { Do. }\end{array}$ \\
\hline \multicolumn{6}{|l|}{ Platinum, Pt } \\
\hline Brazil --_- & 1,000 & $>1,000$ & $>1,000$ & One sample -- & $\begin{array}{l}\text { Noddack and } \\
\text { Ncddack (1931). }\end{array}$ \\
\hline U.S.S.R., Urals _ & 500 & 200 & $>1,000$ & $\begin{array}{l}\text { Eleven sam- } \\
\text { ples. }\end{array}$ & Do. \\
\hline $\begin{array}{l}\text { Platiniridium, (Ir,Pt) } \\
\text { Brazil - }\end{array}$ & 200 & $\ldots-$ & $>1,000$ & One sample -- & Do. \\
\hline $\begin{array}{l}\text { Schreibersite, (Fe,Ni) }{ }_{3} \mathrm{P} \\
\quad \text { Portugal, Sao Juliao de Moreira }\end{array}$ & 1 & 10 & 1.2 & $\begin{array}{l}\text { In meteorite. } \\
\text { Two samples. }\end{array}$ & $\begin{array}{l}\text { Gold rehmidt and } \\
\text { Peters (1932). }\end{array}$ \\
\hline
\end{tabular}

Sulfides, arsenides, selenides, and tellurides

Domeykite, $\mathrm{Cu}_{3} \mathrm{As}$

Mexico, Paracatas

25

$>1,000$

5 One sample -

Noddack and

Noddack (1931).

Argentite, $\mathrm{Ag}_{2} \mathrm{~S}$

Germany, Freiberg

4 Two samples -

Do. 


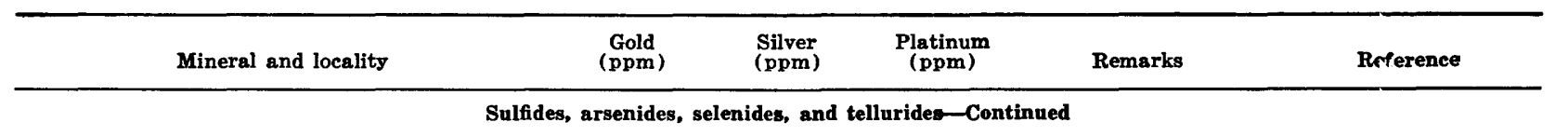

Berzelianite, $\mathrm{Cu}_{2} \mathrm{Se}$

Sweden, Skrikerum

100

$>1,000$

10

One sample --

$\mathbf{5 0 0}$

United States, Butte, Mont

12

Bornite, $\mathrm{Cu}_{5} \mathrm{FeS}_{4}$

Germany, Mansfeld

Galena, PbS

South Africa

Clausthalite, $\mathrm{PbSe}$

Germany, Tilkerode, Harz

Altaite, PbTe

U.S.S.R., Altai

Alabandite, $\mathrm{MnS}$

Roumania, Nagyag, Siebenbürgen_-

Sphalerite, (Zn,Fe)S

Germany:

Chalcopyrite, $\mathrm{CuFeS}_{2}$

Stannite, $\mathrm{Cu}_{2} \mathrm{FeSnS}_{4}$

Czechoslovakia, Zinnwald

Pyrrhotite, $\mathrm{Fe}_{1-\mathrm{xS}}$

Germany, Bühl, near Kassel
Silesia

Bühl, near Kassel

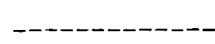

Germany:

Clausthal

Breitenbrunn, near Zwickau

South Africa:

Transvaal, Rustenburg district --

England, Cornwall

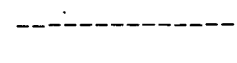

Do

2

5

.2100

Norway, Frфysa

2

.5

1-10

20

800

.2100

1,000

$<.5$

$--\quad<.5$

100

100

.1

.5

.1 Fifteen samples.

Three samples

In basalt

do

do

Do.

Goldscl midt and

Peters (1932).

Noddack and Nodc'ack (1931).

Goldscl midt and

Peter's (1932).

Schneiderhöhn and Moritz (1931).

Noddack and Noddack (1931).

Goldscr midt and Peters (1932).

Do.

Noddac ${ }^{l}$ and Nodd nek (1331).

In basalt

Goldschmidt and Peters (1932).

South Africa, Transvaal

10-100
From Meren- sky Horizon on Schild- padnest, Rustenburg district. Content of other ele- ments: $\mathbf{C u}$, Co about 0.1 percent; Pa, 10-100 ppm; $\mathbf{R u}$, Rh, Ir, 0.1- 1 ppm; 0s, a trace.

\section{Schneiderhöhn} (192S). 
TABLE 2.-Analyses of precious metals in minerals made before 1955-Continued

\begin{tabular}{|c|c|c|c|c|c|}
\hline Mineral and locality & $\begin{array}{c}\text { Gold } \\
\text { (ppm) }\end{array}$ & $\begin{array}{l}\text { Silver } \\
(\mathrm{ppm})\end{array}$ & $\begin{array}{l}\text { Platinum } \\
\text { (ppm) }\end{array}$ & Remarks & Reference \\
\hline
\end{tabular}

Sulfides, arsenides, selenides, and tellurides-Continued

Troilite, FeS

United States, Canyon Diablo, Ariz

Mexico:

Coahuila

Ixtlahuaca

0.5

10

1-5

.2

Chile, Corrizatillo

$$
.5
$$

Esthonia, Tennasilm

5

15

10

5
0.5 In meteorite -

Niccolite, NiAs

Germany:

Eiselben

Klettenberg, Sauerland

Austria, Schladming, Styria

Pentlandite, $(\mathrm{Ni}, \mathrm{Fe}, \mathrm{Co})_{9} \mathrm{~S}_{8}$

Norway, Espedalen

Germany, St. Blasien, Schwarzwald

South Africa, Rustenburg district, Transvaal

Siegenite, $(\mathrm{Co}, \mathrm{Ni})_{3} \mathrm{~S}_{4}$

Germany, Müsen near Siegen

Pyrite, FeSz

Norway:

Setesdalen

Sulitjelma

Italy:

Calceranica

Libiola, Genova Province

Boccheggiano, Grosseto

Chuch e Servette

Pestarena

Lavanchetto

Alfenza
$1 \quad 10-100$

10 .5

6

$.5 \quad 10-100$

1.0

1
1-10

6

.2

.3

- do

do

do

do

do

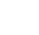

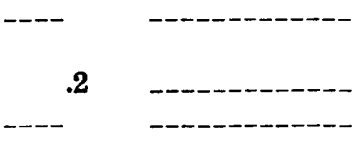

2

.2

5-10

.5

10

70

1.1

.3

200

20

6.5

\section{South Africa:}

Rustenburg district,Transvaal _--

Witwatersrand

\section{5-10}

3

40
Sperrylite, PtAss

Canada, Vermillion mine, Ontario
Two samples -

Noddack and Noddack (1931).

Goldschmidt and

Peters (1932).

Schneiגerhöhn and Moritz (1931).

Goldschmidt and Peters (1932).

Noddack and Nodiack (1931).

Do.

Minguzzi (1947).

$$
\text { Do. }
$$

Do.

Do.

Do.

Do.

Do.
Crystalline

aggregate

contained

$12 \mathrm{ppm} \mathrm{Au}$ and a crystal contained 0.93 ppm Au. Two samples.

10-50 Nickeliferous_

Three samples

One sample --
Schne derhöhn and Mor'itz (1931).

Hegernann and Leybold (1954).

Noddack and Noddack (1931). 
TABLE 2.-Analyses of precious metals in minerals made before 1955-Continued

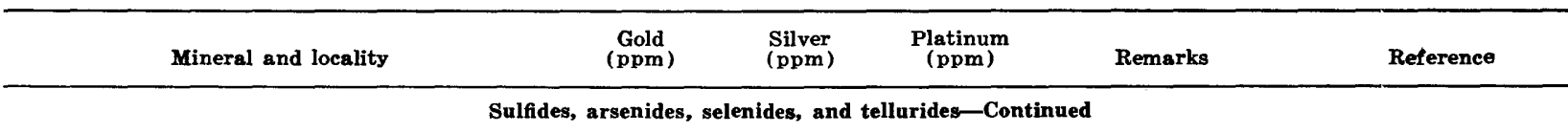

Cobaltite, CoAsS

Canada, Cobalt, Ontario

Norway, Skutterud

5

10

.5

Sweden, Tunaberg

Germany:

Dreikönigsstollen Buchholz, Saxony Glücksbrunn, Altenstein,

Sachsen-Meine

Gersdorffite, NiAsS

Germany:

Müsen, near Siegen

$10-100$

Friedensgrube, Lichtenberg,

Oberfranken

Lobenstein, Vogtland, Thüringen

Michaelis Fundgrube, Triebel, near Zwickau

Ullmannite, NiSbS

Germany:

Müsen, near Siegen

Salchendorf, near Siegen $10-100$

5

$10-100$

20

10
Landeskrone, Willnsdorf, near

Siegen

Safflorite, CoAss

Germany:

Schneeberg, Saxony ------

Dachsberg, near Riechelsdorf -.Rammelsbergite, NiAs 2

Germany, Gessellschafter Zug,

Schneeberg, Saxony

Marcasite, $\mathrm{FeS}_{2}$

Germany, Westphalia

y -.--.-----

Arsenopyrite, FeAsS

Norway, Skutterud

Germany, Ehrenfriedersdorf,

Saxony

Molybdenite, $\mathrm{MoS}_{2}$

United States, Climax, Colo

Australia, Kingsgate, Glenn Innes,

New South Wales

Czechoslovakia, Zinnwald _.........

Germany:

Sadisdorf near Schmiedeberg,

Saxony

Altenberg, Saxony

Norway:

Telemarken

Sörumsassen near Drammen

Råde, Østfold

Undalen
5

200

1

10

10

5

$10-100$

$10-100$

1

10-100

\section{1}
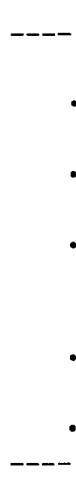

20

$100-$

1,000

.5

10-100

5

$10-100$

.5

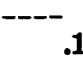

.3

4

5

100

8

90

.5

10

100

100

1-10

.1

5

2

10-100

2

.5

.5

.05

.05
2

One sample --

.5

.1

.1

.1

.1

.1

Gold schmidt and

Peters (1932).

Noddack and

Ncddack (1931).

Gold :chmidt and

Peters (1932).

Do.

Do.

Do.

Do.

Do.

Do.

Do.

Do.

Do.

Do.

Do.

Do.

Do.

$.1 \quad$ One sample --

Nodde $\mathrm{k}$ and

Nod'dack (1931).

.4 Four samples_

Do.

Goldschmidt and Peters (1932).

Do.

Do.

Do.

Do.

Do.

Do.

Do.

Do.

Do. 
TABLE 2.-Analyses of precious metals in minerals made before 1955-Continued

\begin{tabular}{|c|c|c|c|c|c|}
\hline Mineral and locality & $\begin{array}{l}\text { Gold } \\
\text { (ppm) }\end{array}$ & $\begin{array}{l}\text { Silver } \\
\text { (ppm) }\end{array}$ & $\begin{array}{l}\text { Platinum } \\
\text { (ppm) }\end{array}$ & Remarks & Reference \\
\hline
\end{tabular}

Sulfides, arsenides, selenides, and tellurides-Continued

Skutterudite, CoAss

Germany:

Niederrasmstadt near Darmstadt_

$\begin{array}{rrr}0.3 & 1,000 & - \\ 2.7 & 10 & - \\ .1 & 1-5 & - \\ & & \\ .3 & 10-100 & - \\ .5 & 100- & - \\ & 1,000 & \\ 5 & 10 & - \\ .8-.5 & 5 & -\end{array}$

Goldschmidt and

Riechelsdorf, Hessen

Bieber, Hessen

Frauenbreitunger, Saxony-

Meiningen -_.

Schneeberg, Saxony _._._._.

Hasserode, Harz

Austria, Schladming, Styria

$.8-.5$

Sulfosalts

Tetrahedrite, $\mathrm{Cu}_{12} \mathrm{Sb}_{4} \mathrm{~S}_{13}$

England, Cornwall

$\begin{aligned} 8 & >1,000 \\ 60 & >1,000\end{aligned}$

2

$.2 \quad$ Seven samples

800

40

$15>1,000$

90
$>1,000$

\section{Two samples -}

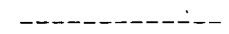

---------- --

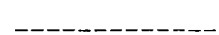

Peter (1932).

Do.

Do.

Do.

Do.

Do.

Enargite, $\mathrm{Cu}_{3} \mathrm{AsS}_{4}$

United States, Butte, Mont

Argyrodite, $\mathbf{A g}_{8} \mathrm{GeS}_{6}$

Bolivia

\section{Halides}

Halite, $\mathrm{NaCl}$

England, Cheshire

0.103

N.D. N.D.

Two samples -

Liversi dge (1897).

Germany, Stassfurt

N.D. N.D.

One sample --

Do.

Sylvite, $\mathrm{KCl}$

Germany, Mecklenburg

.003

N.D

N.D.

do

Friedrirk (1906).

Carnallite, $\mathrm{KMgCl}_{3}: 6 \mathrm{H}_{2} \mathrm{O}$

Germany, Bernburg

$.012 \quad$ N.D. N.D.

do

Do.

Simple oxides

Pyrolusite, $\mathrm{MnO}_{2}$

Czechoslovakia, Platten, Bohemia

Cassiterite, $\mathrm{SnO}_{2}$

Bolivia, Potosi

Indonesia, Bangka Island

Germany, Breitenbrunn near

Zwickau, Saxony _...-

Czechoslovakia, Schönfeld near

Schlaggenwald

South-West Africa:

Sandamab

Nubeb
0.2

$$
4
$$

10

Four samples .

Noddack and

Noddack (1931).

$.5 \quad 10-100$

.5

.5

Three samples

.5

.5

.5
10

$5 \quad 10$

5

5
.5

$\begin{array}{rrr}5 & & \\ 10 & .2 & \\ 1 & .2 & -\ldots-\ldots\end{array}$

Goldscl midt and Peters (1932).

Do.

Do.

Do.

Do. 
TABLE 2.-Analyses of precious metals in minerals made before 1955-Continued

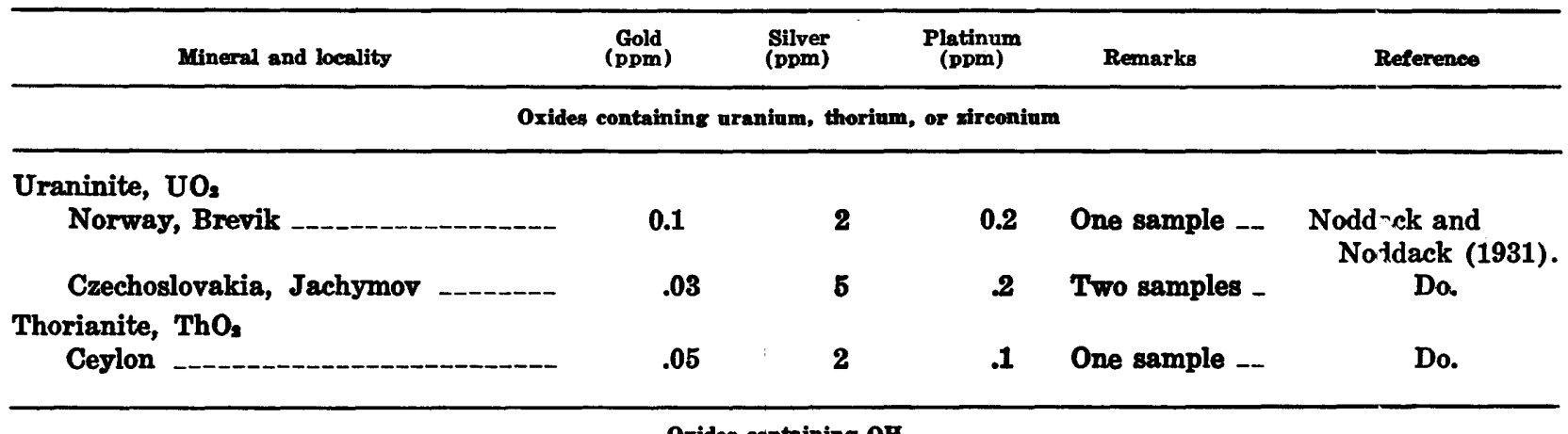

Oxides containing OH

\begin{tabular}{|c|c|c|c|c|c|}
\hline $\begin{array}{l}\text { Psilomelane, }\left(\mathrm{Ba}, \mathrm{H}_{2} \mathrm{O}\right)_{2} \mathrm{Mn}_{5} \mathrm{O}_{10} \\
\text { Germany, } \mathrm{Harz}\end{array}$ & 2 & 50 & 2 & Two samples - & $\begin{array}{l}\text { Noddack and } \\
\text { Noddack (1931). }\end{array}$ \\
\hline
\end{tabular}

Chromite, $(\mathrm{Mg}, \mathrm{Fe})_{2} \mathrm{CrO}$

United States:

Mineral Hills, $\mathbf{P a}$

Lancaster County, Texas, Pa

Norway, Feragen, Bebiet

Hausmannite, $\mathrm{Mn}_{\mathbf{3}} \mathrm{O}_{4}$

Sweden, Lángban
0.2

2

.1

$\begin{array}{rrr}.2 & 1 & 1 \\ .2 & 1 & - \\ .2 & 1 & \cdots\end{array}$

110
Golds'hmidt and

Pet?rs (1932).

Do.

Do.

Multiple oxides containing $\mathrm{Nb}, \mathrm{Ta}$, and $\mathrm{Ti}$

Columbite, ( $\mathrm{Fe}, \mathrm{Mn})(\mathrm{Nb}, \mathrm{Ta})_{2} \mathrm{O}_{0}$

Norway, Arendal

0.05

2

8
Twenty-three samples.

Nodds ck and

Nod dack (1931).

Plagioclase, $(\mathrm{Na}, \mathrm{Ca})\left(\mathrm{Al}, \mathrm{Si}_{2} \mathrm{Si}_{2} \mathrm{O}_{8}\right.$

South Africa, Rustenburg district,

Transvaal

Pyroxenes:

Bronzite, (Mg,Fe)SiO,

South Africa, Rustenburg district,

Transvaal

Diallage, $\mathrm{Ca}(\mathrm{Mg}, \mathrm{Fe}) \mathrm{Si}_{2} \mathrm{O}_{6}$

South Africa, Rustenburg district,

Transvaal

Olivine, (Mg,Fe) ${ }_{2} \mathrm{SiO}_{4}$

South Africa, Rustenburg district,

Transvaal

$$
<0.5
$$

Two samples -

Noddr $\mathrm{ck}$ and

Noc'Jack (1931).

Gadolinite, $\mathrm{Y}_{2} \mathrm{FeBe}_{2} \mathrm{Si}_{2} \mathrm{O}_{0}$

Norway, Iveland

Arandisite, (tin silicate?)

South-West Africa, Arandis

Hellandite, silicate of $\mathrm{Ca}$ and $\mathrm{Y}$

Norway, Kragerö

$<0.5$

$---$

$$
<0.5
$$

$---$

1-5

.5

$--$

1-5

.5

2

.5

5

.1
1.0

2

2

.1 One sample --
Do.

Do.

Schnej derhöhn and Moritz (1931).

\section{Do.}

Nodda $\mathrm{k}$ and

Noddack (1931)

Goldschmict and

Peters (1932).

Noddack and Noddlack (1931). 
TABLE 2.-Analyses of precious metals in minerals made before 1955-Continued

\begin{tabular}{|c|c|c|c|c|c|}
\hline Mineral and locality & $\begin{array}{l}\text { Gold } \\
\text { (ppm) }\end{array}$ & $\begin{array}{l}\text { Silver } \\
\text { (ppm) }\end{array}$ & $\underset{\text { (ppm) }}{\text { Platinum }}$ & Remarks & Reference \\
\hline \multicolumn{6}{|c|}{ Phosphates } \\
\hline $\begin{array}{l}\text { Triplite, }(\mathrm{Mn}, \mathrm{Fe})_{2}\left(\mathrm{PO}_{\mathbf{6}}\right) \mathbf{F} \\
\text { South-West Africa, Sandamab }\end{array}$ & 0.5 & 5 & 0.2 & - - - - - - - - --- & $\begin{array}{l}\text { Goldschmidt and } \\
\text { Peters (1932). }\end{array}$ \\
\hline
\end{tabular}

Nitrates

Soda Niter, $\mathrm{NaNO}_{3}$

Chile

0.110 N.D. N.D. One sample -- Liversidge (1897).

Sulfates

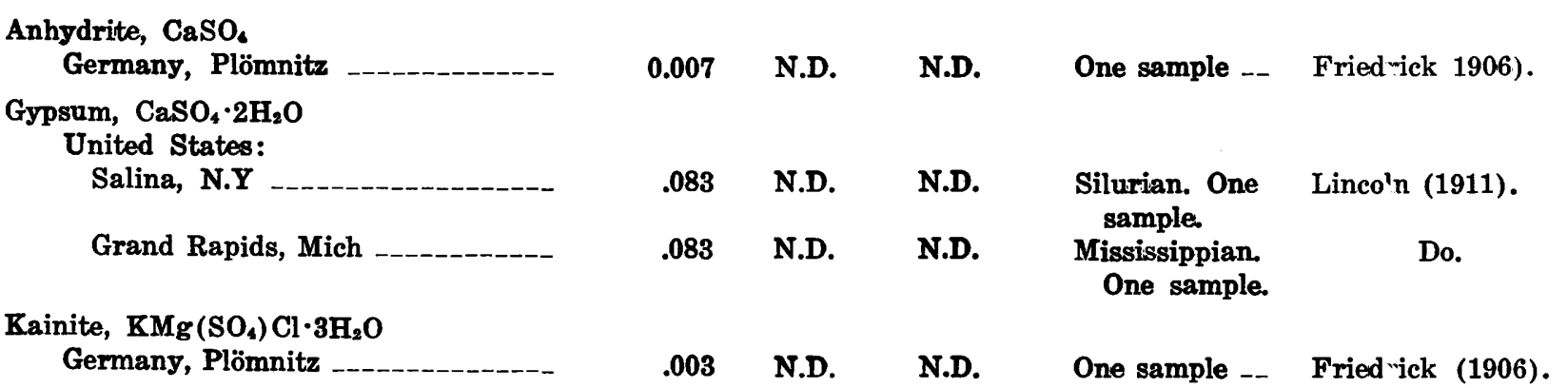

Both Anoshin and Potap'yev (1966) and Shcherbakov and Perezhogin (1964) show that the gold in the quartz exceeds that in the feldspars by a factor of 2.7. Shcherbakov and Perezhogin noted from the analyses of monomineralic fractions of igneous rocks that the average gold content decreases from magnetite and ferromagnesian silicates to feldspars. They reported (table 3) the gold content of the major rock-forming minerals as follows: quartz, 11 ppb (parts per billion); feldspar, 4 ppb; biotite, 4 ppb; muscovite, $3.8 \mathrm{ppb}$; amphibole, $5.9 \mathrm{ppb}$; pyroxene, $16 \mathrm{ppb}$; olivine, $14 \mathrm{ppb}$; and magnetite, $48 \mathrm{ppb}$.

Mantei and Brownlow (1967) have made many neutron activation analyses of minerals from the Marysville quartz diorite stock (table 3). The diorite has an appreciably higher gold content than the average diorite, and most of its component minerals have unusually high gold contents. The reported gold contents increase from quartz and feldspar (65 ppb) to biotite (76 ppb) and reach a maximum in hornblende (100 ppb). The gold content of magnetite was found to be only $37 \mathrm{ppb}$, in contrast to the findings of Shcherbakov and Perezhogin (1964) who report more gold in magnetite than in the silicat? minerals. Mantei and Brownlow accounted for the lower gold content of magnetite, compared. with other minerals they analyzed, by pointing out that the structure of magnetite is relatively closed compared to the structures of biotite and hornblende and that "magnetite may have formed before the silicates, at a time whon the concentration of gold in the magma was fairly low."

Badalov (1965) examined th $\%$ average amounts of gold, silver, selenium, and tellurium in the disseminated copper-molybdenum deposits of the Almalyk district in the U.S.S.R. The sequence of the formation of the predominate minerals in the ores was from earliest to latest: magnetite, molybdemite, pyrite, chalcopyrite, sphalerite, and galena. Pyrite is by far the most abundant mineral and carries $3 \mathrm{ppm}$ gold. Chalcopyrite is the second most abundant mineral and was the chief "concentrator" of gold; it contains $22 \mathrm{rpm}$. Native gold is common, and gold telluridas are rare.

Noddack and Noddack (1931) looked for but did not detect gold in the fol owing minerals: allanite, alvite (variety of zircon), andalusite, aragonite, beryl, brewsterite, bronzite, 


\begin{tabular}{lccc}
\hline \multicolumn{1}{c}{ Mineral and locality } & $\begin{array}{c}\text { Gold } \\
\text { (ppm) }\end{array}$ & Remarks & Reference \\
\hline $\begin{array}{c}\text { Arsenolamprite, As } \\
\text { Germany, Thüringia }\end{array}$ & Elements & \\
Iron, (Fe, Ni) & 5 & $\begin{array}{c}\text { Silver, 1 ppm. Spectographic an- } \\
\text { alysis. }\end{array}$ & Fischer (1958-59). \\
U.S.S.R., Sikhote-Alin' & 1.15 & $\begin{array}{c}\text { One sample of meteorite. In troilite. } \\
\text { 0.067 ppm Au. Neutron activation } \\
\text { analysis. }\end{array}$ & $\begin{array}{c}\text { Shcher-bakov and } \\
\text { Perezhogin } \\
(1961) .\end{array}$ \\
\hline
\end{tabular}

Sulfides

Arsenopyrite, FeAsS

U.S.S.R., Central Chukotki

Germany, Thüringia

200

.5

Chalcopyrite, $\mathrm{CuFeS}_{2}$

U.S.S.R., Almalyk

22

Germany, Thüringia

Cobaltite, CoAsS

Germany, Thüringia

Galena, PbS

U.S.S.R:

Central Chukotki _-_-_-_-_-_ 5

Almalyk

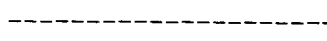

Germany, Thüringia

Molybdenite, $\mathrm{MoS}_{2}$

Germany, Thüringia

Pyrite, $\mathrm{FeS}_{2}$

East Greenland

U.S.S.R :

Almalyk

3

Do

Central Chukotki

Germany, Thüringia
Samples from veins and ore minerals.

Silver, 15 ppm. Spectrographic analysis.

Chalcopyrite is the chief "concentrator" but pyrite is the chief "carrier." Content in parts per million of $\mathrm{Pd}, 0.21, \mathrm{Pt}, 0.02, \mathrm{Ag}$, 0.02 . Analyses by fire assay followed by spectrographic.

Pd, $0.2 \mathrm{ppm}, \mathrm{Ag} 10^{-3}$ percent. Spectrographic analysis.

Silver, 20 ppm. Spectrographic analysis.

Silver, $450 \mathrm{ppm}$. Pd, $0.032 \mathrm{ppm}$. Analyses by fire assay followed by spectrographic.

Silver, 20-100 ppm. Spectrographic analysis.

Content in parts per million of: $\mathbf{R u}$, 0.03, Rh, 0.4, Pd, 0.6, Pt, 0.4, Ag. $>10^{-2}$ percent.

Content in parts per million of: Ag, 60, Se, 40, and Te, 16. Pyrite is the chief "carrier" but chalcopyrite is the chief "concentrator" of gold.

Silver, 36 ppm, Pt, 0.014 ppm. Analyses by fire assay followed by spectrographic.

Samples from veins and ore minerals.

.2 Silver, 5 ppm. Spectrographic analysis.
Sidoro" (1966).

Fischer (1958-59).

Badalc $*$ (1965) and Badalov and Terekhovich $\left(196^{n}\right)$.

Fischer (1958-59).

Do.

Sidoro" (1966).

Badalor and Tere'rhovich (196i)

Fischer' (1958-59).

Do.

Vincent and

Crocket (1960).

Badalo'r (1965).

Badalor and Terel-hovich (1966).

Sidorov (1966).

Fischer (1958-59). 
TABLE 3.-Analyses of gold in minerals made since 1954-Continued

\begin{tabular}{lccc} 
Mineral and locality & $\begin{array}{c}\text { Gold } \\
(\mathrm{ppm})\end{array}$ & Remarks & Reference \\
\hline \multicolumn{2}{c}{ Sulfides-Continued } \\
\hline
\end{tabular}

Pyrrhotite, $\mathrm{Fe}_{1-\mathrm{x}} \mathrm{S}$

East Greenland

U.S.S.R., Central Chukotki

Sphalerite, (Zn, Fe) S

U.S.S.R., Central Chukotki

500

Stibnite, $\mathrm{Sb}_{2} \mathrm{~S}_{3}$

U.S.S.R., Central Chukotki

Germany, Thüringia

-.----o-n-

Troilite, FeS

United States:

Canyon Diablo, Ariz

Sardis, Ga

U.S.S.R., Sikhote-Alin'

.67

Ullmanite, NiSbS

Germany, Thüringia
0.003

2

Analyses by neutron activation

Samples from veins and ore minerals.

20

.9 Silver, 2 ppm. Spectrographic analysis.
Vincent and Cronket (1960).

Sidorov (1966).

Do.

Do.

Fischer (1958-59).

Baedenker (1967).

Do.

One sample of meteorite found in rocks of Miocene age. Analysis by neutron activation.

One sample of meteorite. The iron part of this meteorite contained 1.15 ppm Au.

1 Silver, $10 \mathrm{ppm}$. Spectrographic analysis.

Sulfosalts

Tetrahedrite, $\mathrm{Cu}_{12} \mathrm{Sb}_{4} \mathrm{~S}_{13}$

Germany, Thüringia
0.02 Silver, 20-100 ppm. Spectrographic analysis.
Magnetite, $\mathrm{Fe}_{3} \mathrm{O}_{4}$

United States, Helena, Mont

0.037

U.S.S.R., Altai-Sayan folded belt (?)

Pyrolusite, $\mathrm{MnO}_{2}$

Germany, Thüringia

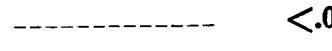

Quartz, $\mathrm{SiO}_{2}$

U.S.S.R.:

Central Chukotki

2

Altai and Transbaikal

Altai-Sayan folded belt (?)
Forty-four samples from the Marysville quartz diorite stock, 20 miles northwest of Helena, Mont. Fifty-one analyses made of these samples which ranged in gold from 0.003 to $0.329 \mathrm{ppm}$. Analyses by neutron activation.

Seven samples. Analyses by neutron activation.

Silver, 3 ppm. Spectrographic analysis.

Samples from veins and ore minerals.

Analyses by neutron activation. $----$
Analysis by neutron activation. Nine samples.

\section{Mantei and Brownlow (19i7).}

Shcherbakov and Per?zhogin (1994).

Fischer (1958-59).

Sidorev (1966).

Anosk in and Potap'yev (1966).

Shcherbakov and Peræzhogin (19:4). 


\begin{tabular}{|c|c|c|c|}
\hline Mineral and locality & $\begin{array}{c}\text { Gold } \\
(\mathrm{ppm})\end{array}$ & Remarks & R ? ference \\
\hline \multicolumn{4}{|c|}{ Oxides - Continued } \\
\hline $\begin{array}{l}\text { Quartz and feldspar } \\
\text { United States, Helena, Mont }\end{array}$ & 0.065 & $\begin{array}{l}\text { Ten samples from the Marysville } \\
\text { quartz diorite stock, } 20 \text { miles } \\
\text { northwest of Helena, Mont. } \\
\text { Twelve analyses made of these } \\
\text { samples which ranged in gold } \\
\text { from } 0.006 \text { to } 0.176 \text { ppm. An- } \\
\text { alyses by neutron activation. }\end{array}$ & $\begin{array}{l}\text { Mante: and } \\
\text { Bror'nlow } \\
\text { (1967). }\end{array}$ \\
\hline $\begin{array}{l}\text { Wad, Mn oxide } \\
\text { Germany, Thüringia }\end{array}$ & $<.01$ & $\begin{array}{l}\text { Silver, } 5 \text { ppm. Spectrographic an- } \\
\text { alysis. }\end{array}$ & Fischer $(1958-59)$. \\
\hline
\end{tabular}

Silicates

Feldspars, $\mathrm{Al}$ silicates with $\mathrm{K}, \mathrm{Na}$, and

$\mathrm{Ca}$

U.S.S.R., Altai-Sayan folded belt (?)

Altai and Transbaikal

Microcline, $\mathrm{KAlSi}_{3} \mathrm{O}_{8}$

U.S.S.R., S. E. Altai

Hornblende, $\mathrm{Ca}_{2} \mathrm{Na}\left(\mathrm{MgFe}^{+2}\right)_{4} \mathrm{Al}$,

$\left.\mathrm{Fe}^{+3} \mathrm{Ti}\right)_{3} \mathrm{Si}_{8} \mathrm{O}_{22}(\mathrm{O}, \mathrm{OH})_{2}$

United States, Helena, Mont.

Pyroxene, silicates of $\mathrm{Ca}, \mathrm{Mg}, \mathrm{Fe}$, and others

U.S.S.R., Altai-Sayan folded

belt (?)

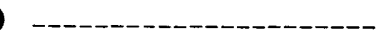

Tourmaline, complex silicate of B and

Al

U.S.S.R., Altai-Sayan folded

belt (?)

Muscovite, $\mathrm{KAl}_{2}\left(\mathrm{AlSi}_{3}\right) \mathrm{O}_{10}(\mathrm{OH})_{2}$

U.S.S.R., Altai-Sayan folded

belt (?)
.0003 Analysis by neutron activation.

.018 One sample from a migmatite. Analysis by neutron activation.

.0059 Fourteen samples analyzed by neutron activation method.

.077 Two samples analyzed by neutron activation.

0.0040 Twenty-seven samples. Analyses by neutron activation.

.100 Thirty-seven samples from the Marysville quartz diorite stock, 20 miles northwest of Helena, Mont. Forty-two analyses made of these samples which ranged in gold from 0.003 to $0.823 \mathrm{ppm}$. Analyses by neutron activation.

.016 Eight samples analyzed by neutron activation.

.012 Four samples analyzed by neutron activation.

.0038 Seven samples analyzed by neutron activation.
Shcherhakov and Perezhogin (1964).

Anoshin and Potao'yev (1966).

Shcherbakov and Pererhogin (1964).

\section{Do.}

Do.

\section{Mantei and Brownlow (1967).}

Shchertakov and Perezhogin

(1964). 
TABLE 3.-Analyses of gold in minerals made since 1954-Continued

\begin{tabular}{ccc}
\hline Mineral and locality & $\begin{array}{c}\text { Gold } \\
(\mathbf{p p m})\end{array}$ & Remarks \\
\hline Silicates-Continued & Reference \\
\hline
\end{tabular}

Biotite, $\mathrm{K}(\mathrm{Mg}, \mathrm{Fe})_{3}\left(\mathrm{AlSi}_{3}\right) \mathrm{O}_{10}(\mathrm{OH})_{2}$

United States, Helena, Mont

U.S.S.R., Altai-Sayan folded

belt (?)

Altai

.0091

.0040

Eight samples analyzed by neutron activation. Marysville quartz diorite stock, 20 miles northwest of Helena, Mont. Fifty-three analyses made of these samples which ranged in gold from 0.002 to $0.924 \mathrm{ppm}$. Analyses by neutron activation.

hree samples from granite which were analyzed by neutron activation. activation.

.0039 Two samples analyzed by neutron
activation.

.0039 Two samples analyzed by neutron
activation.

Sphene, CaTiSiOs

U.S.S.R., Altai-Sayan folded

belt (?)
Mantei and

Browslow (1967).

Shchertakov and Perezhogin

(1964).

Do.
Shcherk akov and Perezhogin (1964). cristobalite, daubreelite (from meteorites), diopside, epidote, euclase, garnet, gersdorffite, harmotome, hauerite, heulandite, hornblende, kaolin, lepidolite, leucite, malachite, malacon, molybändocker [ilsemannite], molybdosodalite, muscovite, nepheline, olivine, orpiment, orthoclase, pyroxene, rhodochrosite, rutile, serpentine, stibnite, tantalite, thalenite, and thortveitite. The lower limit of detection of the analytical method they used seems to be about $10 \mathrm{ppb}$. Other specimens of some of these minerals analyzed by neutron activation methods also show less than $10 \mathrm{ppb} \mathrm{Au}$ (table 3).

Platinum exceeds gold in meteorites, as well as in metasilicate minerals and orthosilicate minerals.

\section{COMPOSITION AND THE FINENESS OF GOLD}

Samples of native gold from 48 places throughout the world have been analyzed for selected elements (Gay, 1963). The frequency of occurrence of 30 elements detected (in order of frequency) is as follows:
Detected in 90-100 percent of the samples _.

Detected in 18.8-37.6 percent of the samples $\mathrm{Pb}, \mathrm{Ti}, \mathrm{Al}, \mathrm{Sb}, \mathrm{Hg}$, V, Bi, Mn, Si, As, Sn

Detected in 6.3-14.6 percent of the samples $\mathrm{Mg}, \mathrm{Ni}, \mathrm{Ca}, \mathrm{Zn}$, Pd, Pt. Te

Detected in 2.1-4.2 percent of the samples $\mathrm{B}, \mathrm{Co}, \mathrm{Cr}, \mathrm{Mo}, \mathrm{Cd}$, Rh, Sr, W, Zr

It is probable that not all the elements in the foregoing list were looked for by most analysts.

Warren and Thompson (1944) studied the composition of 66 samples of native gold, about 75 percent of which originated ir Canada. All samples contained silver, copper, and iron. The number of occurrences of the various other elements in the gold were: titanium. 52; mercury, 42; manganese, 40; lead, 37; vanadium, 28; tin, 22; antimony, 21; bismuth, 19; arsenic, 17; zinc, 15; cadmium, 14; tellurium, 8; platinum, 3; and palladium, 2. Silver wis usually present in amounts exceeding 0.5 percent and 
copper was usually present in amounts from 0.1 percent to 0.5 percent. The remaining elements, when present in the gold, were usually in amounts of less than 1 percent.

Wise (1964), in a study of binary alloys of gold, gives the various maximum high-temperature solid solubilities of elements in gold as follows:

100 percent
46 percent $\mathrm{Ag}, \mathrm{Cu}, \mathrm{Ni}, \mathrm{Pd}, \mathrm{Pt}$
$21.5 \sim 19$ percent $\mathrm{Cd}, \mathrm{Cr}, \mathrm{Hg}$
13 percent
$10.9-7.7$ percent $\mathrm{Mn}, \mathrm{Ta}, \mathrm{Co}, \mathrm{In}$
$5.2-1.2$ percent $\mathrm{Ga}, \mathrm{Sn}, \mathrm{Mg}, \mathrm{Al}$
$\mathrm{Ti}, \mathrm{Ge}$
$<1$ percent $\mathrm{As}, \mathrm{Bi}, \mathrm{Ca}, \mathrm{Mo}, \mathrm{Pb}$,
$\mathrm{Pr}, \mathrm{Rh}, \mathrm{Sb}, \mathrm{Th}$,
$\mathrm{Tl}, \mathrm{U}$, and per-
haps others.

The fineness of gold, and especially its relation to the genesis of gold denosits, has been studied by numerous workers (Gay, 1963; Sundell, 1936; Mertie, 1940). Fineness refers to the ratio of gold to the sum of the gold plus the silver in the naturally occurring alloys and is defined as 1,000 times $\mathbf{A u} /(\mathbf{A u}+\mathbf{A g})$.

Mertie (1940) noted that pure gold has not been found in nature, but that gold is always alloyed with silver and a small amount of the base metals such as iron and copper. The purest gold reported by Mertie was from the Great Boulder mine, in the Kalgoorlie district of Western Australia; it was 999.1 fine. Mertie concluded that fineness is rarely less than 600 and is generally never less than 400 , although Lincoln (1911) reported a fineness of 246 for silver-gold alloys in some mafic igneous rocks.

The color of gold in polished section is an index of its fineness, according to Eales (1961). Nearly pure gold has a golden color with a ruby tint. With increasing amounts of silver, the color changes to yellow and eventually becomes a pale silvery yellow color as in electrum. Others have noted color differences in gold due to variations in fineness (Mather, 1937 ; Russell, 1929; Edwards, 1958).

Boyle (1960), Ward (1958), and Lincoln (1911) have commented on the fineness of gold in different country rocks. Boyle thought that the fineness of gold in lode deposits reflected the nature of the country rock and that this seemed to indicate that the deposits were formed by diffusion of gold and silver through the country rock. For instance, in the Yellowknife district of Canada, gold in deposits in greenstone has a gold-silver ratio of 5:1 (= fineness 833), wherras gold in quartz lenses in sedimentary rocks has a ratio of 3.5:1 (= fineness 778). Ward observed that gold in some ore bodies in Western Australia that are genetically related to albite porphyry intrusives has a gold to silver ratio greater than 9:1 $(=$ fineness 9( $)$ ). Lincoln noted that the fineness of gold $i$ higher in silicic igneous rocks than in maf? varieties; gold in silicic igneous rocks aver ces 979 in fineness, that in intermediate typ?s 451 , and that in mafic types $\mathbf{2 4 5}$.

Native gold at the surface and in the oxidized zone of a mineral deposit is usuall" finer than is the native gold in the unoxidize ore (Don, 1898; Fisher, 1945; Colin, 1946; MacGregor, 1928; Mackay, 1944; Mills, 1954. and Gay, 1963). Below the oxidized zone, hc rever, gold fineness seems to be largely independent of depth (Gay, 1963). A small decrase in the fineness of gold with depth in the Lily mine, Republic of South Africa, is reported by Anhaeusser (1966) and shown in table 4. On the other hand, average gold fineness in the Zwartkopje shoot in the Sheba mine, Republic of South Africa, increases with depth from the 14 level (about 910 fine) to the 26 level (about 950 fine) within a vertical distance of about 550 feet (Gay, 1964).

TABLE 4.-Variations in fineness of gold with depth, Lily Mine, Transvaal, South Africa

[After Anhaeusser, 1966]

\begin{tabular}{|c|c|c|c|c|}
\hline Level & $\begin{array}{c}\text { Depth } \\
\text { in feet } \\
\text { below } \\
2,600-\text {-foot } \\
\text { datum } \\
\text { plane }\end{array}$ & (percent) & $\underset{\text { (percent) }}{\text { Silvor }}$ & Fineness \\
\hline $\begin{array}{l}\text { 70-foot } \\
1 \\
11 / 2 \\
2\end{array}$ & 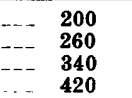 & $\begin{array}{l}91.50 \\
91.15 \\
90 . \backsim 8 \\
89.04\end{array}$ & $\begin{array}{r}8.50 \\
8.85 \\
972 \\
10.96\end{array}$ & $\begin{array}{l}915 \\
911.5 \\
903 \\
890\end{array}$ \\
\hline
\end{tabular}

Gold fineness was noted by Gay (1963) to vary from east to west in some Witwatersrand deposits as follows: 865.8, 884.0, 870.6, 926.0, 912.0, 924.0, and 970.0. Sharwood (1911) reports some lateral variation in fineness of bullion from mines in the Lead district, South Dakota; as shown in table 5, the range in fineness is small. 
TABLE 5.-Fineness, in percent, of mill bullion prior to 1882 at the Homestake mine, South Dakota

[Sharwood, 1911]

\begin{tabular}{|c|c|c|c|c|}
\hline Mine & Gold & Silver & $\begin{array}{c}\text { Base } \\
\text { metal }\end{array}$ & Fineness \\
\hline $\begin{array}{l}\text { Homestake } \\
\text { Highland } \\
\text { Terra } \\
\text { Deadwood } \\
\text { De Smet }\end{array}$ & $\begin{array}{c} \\
- \\
-\end{array}$ & $\begin{array}{l}17.0 \\
15.5 \\
16.0 \\
14.0 \\
17.0\end{array}$ & $\begin{array}{l}1.0 \\
1.5 \\
1.5 \\
1.0 \\
1.0\end{array}$ & $\begin{array}{l}828 \\
843 \\
838 \\
859 \\
828\end{array}$ \\
\hline
\end{tabular}

Lateral variation in fineness is shown by analyses of the bullion from nine representative mines in Gilpin County, Colo. (Collins, 1902). During the period 1870 to 1880 , the fineness ranged from mine to mine from $\mathbf{7 5 3}$ to 897 , and during the period 1880 to 1890 , from 716 to 894 . The average fineness of all the bullion from these mines changed little during the two periods; it was 799 for the first and 789 for the second.

Eales (1961) studied the silver content of gold from four hydrothermal deposits in Southern Rhodesia. Gold that mineragraphic studies show to have crystallized early, and that is enclosed in chalcopyrite and sphalerite, contains more silver than does gold that crystallized later.

The fineness of gold varies directly with particle size in some deposits and inversely with particle size in others (Gay, 1963).

For ore deposits in general, the fineness of the contained gold increases as the grade of ore increases; (Eales, 1961; Lawn, 1924; MacGregor, 1928; and Mackay, 1944).

Fisher (1950) concluded that fineness used with other criteria furnishes a sensitive and reliable guide to the relative temperature of ore formation, at least within the epithermal and the upper part of the mesothermal range of temperatures. The fineness of epithermal gold is from 500 to 700 . Near the bottom of the epithermal zone (corresponding to the leptothermal zone of Graton, 1933), the fineness is about 700 and may be as much as 800. The fineness of mesothermal gold varies from 750 to 900 , with $850-870$ being common. The fineness of hypothermal gold is always greater than 800 . Fineness of 900 or more results from oxidation under conditions favoring the removal of silver.

Gay (1963) observed that commonly the fineness of placer gold increases downstream from its source. This increase is explained by leaching of the silver and redeposition of the gold. Shcherbina (1956) explains that the high ratio of silver to gold, 37.: (Mason, 1952) in sea waters, compared to only about 10 (Vinogradov, 1956) for the lithosphere as a whole, is an indication of the gre ter mobility of silver.

Mertie (1940) says that in some Alaskan paystreaks the fineness of gold increases downstream from its source, but in others the fineness changes either erratically or not at all. He cites an Alaskan placer gold doposit that was derived from lodes in Tertiary quartz monzonite. The source lodes were being actively mined around 1940, and information on the fineness of both lode and placer gold indicates that the fineness does not increase progressively downstream.

Fineness varies not only from gre in to grain but also within grains (Gay, 1963). Some grains are finer in the center than on the surface (Head, 1935), although Esles (1961) has noted the reverse. Gold extracted by McConnell (1907) from the outer surface of a nugget assayed 60-70 parts per tho'sand finer than gold from the inside of the rugget, and his findings have been cited as evidence that surface waters dissolve an appreciable amount of silver from alluvial gold. However, the difference could have been due to the original character of the primary lode gold or to surficial enrichment of the gold in the zone of oxidation before it was freed (Mertie, 1940). Very fine grained placer gold is usually finer than the coarse gold (Hite, 193?; Sundell, 1936; Fisher, 1945; and Colin, 1946). However, Mertie (1940) reports that in any one paystreak, and at any one place in the paystreak, the reverse is usually true.

\section{REFERENCES CITED}

Anhaeusser, C. R., 1966, Supergene golt enrichment in the Barberton Mountain Land with particular reference to the Lily Mine: Witwatersrand Univ. Econ. Geology Research Unit Inf. Circ. 29, 16 p.

Anoshin, G. N., and Potap'yev, V. V., 1966, Gold in granites of the Kolyvan' (Altay) ard KhangilayShilinskiy (Transbaikalia) massifs (according to radioactivation analysis data): Geokhimiya, no. 9, p. 1070-1074 (in Russian). Translation in Geochemistry Internat., 1966, v. 3, no. 5, F. 850-854.

Badalov, S. T., 1965, On the role of predominant components in the geochemistry of minor and rare elements of ore deposits: Geochemistry Internat., v. 2, no. 5, p. 857-860. 
Badalov, S. T., and Terekhovich, S. L., 1966, Geachemistry of elements of the $\mathrm{Pt}$ group in the Almalk ore region: Akad. Nauk SSSR Doklady, v. 168, p. 1397-1399 (in Russian).

Baedecker, P. A., 1967, The distribution of gold and iridium in meteoritic and terrestrial materials: U.S. Atomic Energy Comm. [Pub.] ORO-267017, and $\mathrm{Ph}$. D. thesis, Kentucky Univ., $110 \mathrm{p}$.

Boyle, R. W., 1960, The geology, geochemistry and origin of the gold deposits of the Yellowknife district: Canada Geol. Survey Mem. 310, 193 p.

Cloke, P. L., and Kelly, W. C., 1964, Solubility of gold under inorganic supergene conditions: Econ. Geology, v. 59, p. 259-270.

Colin, L. L., 1946, Gold fineness in relation to geology; considerations of the Macequece field [Mozambique]: South Africa Mining and Eng. Jour., v. 57, pt. I, p. 279-283.

Collins, G. E., 1902, The relative distribution of gold and silver values in the ores of Gilpin County, Colorado: Inst. Mining and Metallurgy Trans. (London) v. 12, p. 480-499.

Don, J. R., 1898, The genesis of certain auriferous lodes: Am. Inst. 'Mining Engineers Trans., v. 27, 564-668.

Eales, H. V., 1961, Fineness of gold in some Southern Rhodesian gold mines: Inst. Mining and Metallurgy Trans., Bull. no. 660, v. 71, p. 49-73.

Edwards, A. B., 1958, The mineral composition of the Maude and Yellow Girl gold ore, Glen Wills, Victoria: F. L. Stillwell Anniversary Volume Melbourne, Australasian Inst. Mining and Metallurgy p. 105-132.

Fischer, Karl-Wilhelm, 1958-59, Zur Geochemie der Edelmetalle. Spektralanalytische Untersuchungen and Thüringer Gesteinen und Mineralien: Wiss. Zeitschr. Hochschule f. Architekur u. Bauwesen Weimar, v. 6, no. 2, p. 85-91.

Fisher, N. H., 1945, The fineness of gold, with special reference to the Morobe gold field: Econ. Geology, v. 40 , p. $449-495$ and $537-563$.

1950, Application of gold fineness to the search for ore: Australasian Inst. Mining and Metallurgy Proc. 156-157, p. 185-190.

Friedrick, K., 1906, Untersuchungen über den Goldgehalt von Gebirgsproben and Solen deutscher Salzlagerstätten: Metallurgie, v. 3, no. 2, p. 627630.

Gay, N. C., 1963, A review of the geochemical characteristics of gold in ore deposits: Witwatersrand Univ. Econ. Geology Research Unit Inf. Circ. 12, $70 \mathrm{p}$.

1964, The composition of gold from the Barberton mountain land: Witwatersrand Univ. Econ. Geology Research Unit Inf. Circ. 19, 53 p.

Goldschmidt, V. M., and Peters, Cl., 1932, Zur Geochemie des Edelmetalle: Gesell. Wiss. Göttingen, Nachr., Math-Phys. Kl. no. 4, p. 377-401.

Goni, J., Guillemin, C., and Sarcia, C., 1967, Géochemie de l'or exogène. Étude expérimentale de la formation des dispersions colloidales d'or et de leur stabilité: Mineralium Deposita, v. 1, p. 259-268.

Graton, L. C., 1933, The depth-zones in ore deposition: Econ Geology, v. 28, p. 513-555.

Head, R. E., 1935, Form and occurrence of gold in pyrite-coated gold: Canadian Minir? Jour., v. 56, (II), p. 517-521.

Hegemann, Friedrich, and Leybold, Chr., 1954, Eine Methode zur quantitative spektrochemischen Analyse von Pyrite: Zeitschr. Erzbergbau u. Metallhüttenwesen, v. 7, p. 108-113.

Helgeson, H. C., and Garrels, R. M., 1968, Hydrothermal transport and deposition of gold: Econ. Geology, v. 63, p. 622-635.

Hite, T. H., 1933, Fine gold and platinum of Snake River, Idaho: Econ Geology, v. 28, p. 256-265.

Krauskopf, K. B., 1951, The solubility of gold: Econ. Geology, v. 46, p. 858-870.

Lawn, J. G. E., 1924, Presidential address on the subject of silver in Witwatersrand ores: Geol. Soc. South Africa Proc., v. 27, p. 19-31.

Lincoln, R. C., 1911, Certain natural assxiations of gold: Econ. Geology, v. 6, p. 247-302.

Liversidge, A., 1897, Presence of gold in natural saline deposits and marine plants: Tour. Chem. Soc., v. 71, p. 298-299.

McConnell, R. G., 1907, Report on the gold values in the Klondike high-level gravels: Crnada Geol. Survey, no. 979, $134 \mathrm{p}$.

MacGregor, A. M., 1928, The geology of the country around the Lonely Mine, Bubi District: Geol. Survey Southern Rhodesia Bull., no. 11, 96 p.

Mackay, R. A. C., 1944, Purity of native gold as a criterion for secondary enrichment: Econ. Geology, v. 39, p. 56-68.

Mantei, E. J., and Brownlow, A. H., 1967, Variation in gold content of minerals of the Marysville quartz diorite stock, Montana: Geochim. et Cosmochim. Acta, v. 31, no. 2, p. 225-235

Mason, Brian, 1952, Principles of geochemistry: New York, John Wiley and Sons, 276 p.

Mather, W. B., 1937, Geology and par genesis of gold ores of the Howey mine, Red Lal 3 , Ontario: Econ. Geology, v. 32, p. 131-153.

Mertie, J. B., Jr., 1940, Placer gold in Alarka: Washington Acad. Sci. Jour., v. 30, p. 93-124.

Mills, J. W., 1954. Vertical zoning at the O'Brien gold mine, Kewagama, Quebec: Econ. Geology, v. 49 , p. 423-430.

Minguzzi, Carlo, 1947, Dosatura spettrografica dell oro in piriti italiane: Soc. Toscana Sci. Nat. Atti., Mem., v. 54, p. 210-243.

Noddack, Ida, and Noddack, Walter, 1931. Die Geochemie des Rheniums: Zeitschr. Phys. Chemie, v. 154A, p. 207-244.

Ottemann, J., and Augustithis, S. S., 1967, Goochemistry and origin of "platinum-nuggets" in lateritic covers from ultrabasic rocks and birbirites of W. Ethiopia: Mineralium Deposita, v. 1, p. 269277. 
Palache, Charles, Berman, Harry, and Frondel, Clifford, 1944, Dana's System of Mineralogy: 7th ed., New York, John Wiley and Sons, v. 1, 834 p.

Ramdohr, Paul, 1967, The wide-spread paragenesis of ore minerals originating during serpentinization: Geologiya Rudnykh Mestorozhdenii, no. 2, p. 32 43 (in Russian).

Ringwood, A. E., 1955, The principles governing trace element distribution during magmatic crystallization: Geochim. et Cosmochim. Acta, v. 7, p. 189-202, 242-254.

Russell, A., 1929, On the occurrence of native gold at Hope's Nose, Torquay, Devonshire: Mineralog. Mag., v. 22, p. 159-162.

Schneiderhöhn, H., 1929, The mineragraphy and spectrography of the sulfide platinum ores of the Bushveld complex, chap. XVII, p. 206-246, in Wagner, P. A., The platinum deposits and mines of South Africa: Edinburgh and London, Oliver and Boyd.

Schneiderhöhn, H., and Moritz, H., 1931, Spektrographische Untersuchungen über die Verteilung der Platinmetalle in den Mineralien der südafrikanischen Plantinlagerstätten: Festschr. d. Platinschmelze G. Siebert, Hanau, p. 257-287.

Schwartz, G. M., 1944, Host minerals of native gold: Econ. Geology v. 39, p. 371-411.

Sharwood, W. T., 1911, Analyses of rocks and minerals from Homestake mine, Lead, South Dakota: Econ. Geology, v. 6, p. 729-789.
Shcherbakov, Yu. G., and Perezhogin, G. A., 1964, Geochemistry of gold: Geochemistry Internat., no. 3, p. 489-496.

Shcherbina, V. V., 1956, The geochemical significance of the quantitative silver/gold ratio: Geokhimiya, no. 3, p. 65-83 (in Russian).

Sidorov, A. A., 1966, Gold-silver mineralization of Central Chukotki: Akad. Nauk SSSR Magadan. Severo-Vostoch. kompleks. Nauchno.-Issled. Inst. Trudy, vyp. 14, 146 p. (in Russian).

Sundell, I. G., 1936, Fineness and composition of alluvial gold from the Ivalojoki, Finnish Lapland: Comm. Geol. Finlande, Bull. no. 115, p. 155-160.

Terziev, G., 1966, Kostovite, a gold-copper telluride from Bulgaria: Am. Mineralogist, v. 51, p. 29-36.

Vincent, E. A., and Crocket, J. H., 1960, Studies on the geochemistry of gold. I. The distribution of gold in rocks and minerals of the Skaergaard intrusion, East Greenland: Geochim. et Cosmochim. Acta, v. 18, p. 130-142.

Vinogradov, A. P., 1956, Regularity of distribution of chemical elements in the earth's crust: Geokhimiya, translation, no. 1, p. 1-43.

Ward, H. J., 1958, Albite porphyries as a guide to gold ore: Econ. Geology, v. 53, p. 754-756.

Warren, H. V., and Thompson, R. M., 1944, Minor elements in gold: Econ. Geology, v. 39, p. 457471.

Wise, E. M., 1964, Gold: recovery, properties, and applications: New York, D. Van N'ostrand, Co., Inc., 367 p. 
